\title{
OSCILLATION AND GLOBAL ATTRACTIVITY IN A DISCRETE SURVIVAL RED BLOOD CELLS MODEL
}

Abstract. We consider the discrete survival red blood cells model

$$
N_{n+1}-N_{n}=-\delta_{n} N_{n}+P_{n} e^{-a N_{n-k}},
$$

where $\delta_{n}$ and $P_{n}$ are positive sequences. In the autonomous case we show that $(*)$ has a unique positive steady state $N^{*}$, we establish some sufficient conditions for oscillation of all positive solutions about $N^{*}$, and when $k=1$ we give a sufficient condition for $N^{*}$ to be globally asymptotically stable. In the nonatonomous case, assuming that there exists a positive solution $\left\{N_{n}^{*}\right\}$, we present necessary and sufficient conditions for oscillation of all positive solutions of $(*)$ about $\left\{N_{n}^{*}\right\}$. Our results can be considered as discrete analogues of the recent results by Saker and Agarwal [12] and solve an open problem posed by Kocic and Ladas [8].

1. Introduction. Most dynamic population evolution models are described by delay differential equations. Analytically, differential delay equations are difficult to handle and therefore many articles have examined difference equations as models. In order to describe the survival red blood cells in animals Ważewska-Czyżewska and Lasota [14] proposed the equation

$$
N^{\prime}(t)=-\delta N(t)+P e^{-a N(t-\tau)} .
$$

Here $N(t)$ is the number of red blood cells at time $t, \delta$ is the rate of death of the cells, $P$ and $a$ describe the generation of the cells per unit time, and $\tau$ is the time needed to produce blood cells. For oscillation of (1.1) we refer to the monograph [4]. Very recently, Saker and Agarwal [12] considered (1.1) with periodic coefficients and studied its oscillation and global attractivity.

2000 Mathematics Subject Classification: 39A10, 92D25.

Key words and phrases: oscillation, global attractivity, discrete survival red blood cells model. 
We remark that in recent years discrete population dynamics has become a very popular subject. In fact, several different models have been studied in $[1-4,6-11,15-16]$. Following this trend, in this paper we shall consider the discrete survival red blood cells model [8]

$$
N_{n+1}-N_{n}=-\delta N_{n}+P e^{-a N_{n-k}},
$$

where

$$
P, a \in(0, \infty), \quad \delta \in(0,1), \quad n \in \mathbb{N} .
$$

The state variable $N_{n}$ in (1.2) represents the number of mature red blood cells in cycle $n$ as a closed system of the mature cells surviving from previous cycles plus the cells that have survived from the previous $k$ cycle. In particular, $P e^{-a N_{n-k}}$ represents the number of mature cells that were produced in the $(n-k)$ th cycle and survived to maturity in the $n$th cycle.

By a solution of (1.2), we mean a sequence $\left\{N_{n}\right\}$ which is defined for $n \geq-k$ and which satisfies (1.2) for $n \geq 0$. Together with (1.2) we consider the initial condition

$$
N_{i}=a_{i} \quad \text { for } i=-k, \ldots, 0 .
$$

Then it is easily seen that the initial value problem (1.2), (1.4) has a unique positive solution $\left\{N_{n}\right\}_{n=1}^{\infty}$.

The variation of the environment plays an important role in many biological and ecological dynamical systems. In particular, the effects of a periodically varying environment are important for evolutionary theory as the selective forces in systems in a fluctuating environment differ from those in a stable environment. Thus, the assumption of periodicity of the parameters in the system (in a way) incorporates the periodicity of the environment (e.g., seasonal effects of weather, food supplies, mating habits, etc.). A modification of (1.2) according to the environmental variation is given by the nonautonomous delay difference equation

$$
N_{n+1}-N_{n}=-\delta_{n} N_{n}+P_{n} e^{-a N_{n-k}},
$$

where

$$
\delta_{n} \text { and } P_{n} \text { are positive sequences. }
$$

We will consider (1.5) together with the initial condition

$$
N_{i}=a_{i} \quad \text { for } i=-k, \ldots, 0 .
$$

Again, it is clear that the initial value problem (1.5), (1.7) has a unique solution $\left\{N_{n}\right\}_{n=1}^{\infty}$.

The plan of this paper is as follows: In Section 2, for the autonomous case we first show that there exists a unique positive steady state $N^{*}$ of (1.2), and prove that (1.2) with (1.3) has a positive solution which is bounded and persistent; we also provide sufficient conditions for the oscillation of all 
positive solutions about $N^{*}$, and when $k=1$ we present sufficient conditions for the global attractivity of $N^{*}$. In Section 3, for the nonautonomous case, assuming that (1.5) has a positive solution $\left\{N_{n}^{*}\right\}$, we present necessary and sufficient conditions for the oscillation of all positive solutions of (1.5) about that solution. Our results can be considered as discrete analogues of the results in [12] and solve an open problem posed by Kocic and Ladas [8, open problem 4.6.1].

2. Autonomous case. In this section we study the oscillatory and asymptotic behavior of positive solutions of (1.2) when $N_{-k}, N_{-k+1}, \ldots, N_{0}$ are nonnegative constants.

Let $I$ be an interval of positive real numbers, and let $f: I \times I \rightarrow I$ be a continuous function. Consider the difference equation

$$
N_{n+1}=f\left(N_{n}, N_{n-k}\right), \quad n=1,2, \ldots
$$

We say that $N^{*}$ is an equilibrium of (2.1) if

$$
N^{*}=f\left(N^{*}, N^{*}\right) \text {, }
$$

that is, the constant sequence $\left\{N_{n}\right\}_{n=-k}^{\infty}$ with $N_{n}=N^{*}$ for all $n \geq-k$ is a solution of (2.1).

Now, we prove that (1.2) has a unique equilibrium $N^{*}$. Observe that the equilibrium points of (1.2) are the solutions of the equation

$$
N^{*}=P e^{-a N^{*}}+(1-\delta) N^{*} .
$$

Set

$$
f(x)=P e^{-a x}+(1-\delta) x-x=P e^{-a x}-\delta x .
$$

Then $f(0)=P>0$ and $f(\infty)=-\infty$, so there exists $x^{*}>0$ such that $f\left(x^{*}\right)=0$. Now since $f^{\prime}(x)=-a P e^{-a x}-\delta<0$ for all $x>0$ we have $f^{\prime}\left(x^{*}\right)<0$, from which it follows that $f(x)=0$ has exactly one solution $x^{*}$, and then (1.2) has a unique equilibrium point $N^{*}$.

We say that a sequence $\left\{N_{n}\right\}$ is bounded and persistent if there exist positive constants $C$ and $D$ such that

$$
C \leq N_{n} \leq D \quad \text { for } n=0,1, \ldots
$$

A difference equation

$$
x_{n+1}=F\left(x_{n}, x_{n-1}, \ldots, x_{n-k}\right)
$$

is said to be permanent if there exist numbers $C$ and $D$ with

$$
0<C \leq D<\infty
$$

such that for any initial conditions $x_{-k}, x_{-k+1}, \ldots, x_{0} \in(0, \infty)$ there exists a positive integer $n_{1}$ which depends on the initial conditions such that

$$
C \leq x_{n} \leq D \quad \text { for } n \geq n_{1} .
$$


The importance of the permanence for biological systems was thoroughly reviewed by Hutson and Schmitt [5].

In the following, we show that every solution of (1.2) is bounded and persistent.

Theorem 2.1. Assume that (1.3) holds and assume that the initial conditions $N_{-k}, N_{-k+1}, \ldots, N_{0}$ are nonnegative constants. Then the solution $\left\{N_{n}\right\}_{n=1}^{\infty}$ is positive, bounded and persistent.

Proof. First assume that $N_{-k}, N_{-k+1}, \ldots, N_{0}$ are nonnegative constants. Then

$$
N_{n+1}=(1-\delta) N_{n}+P e^{-a N_{n-k}}>0,
$$

which proves the nonnegativity result by induction. Now assume that $\left\{N_{n}\right\}$ is not bounded. Then there exists a subsequence $\left\{N_{n_{i}}\right\}$ such that $\lim _{i \rightarrow \infty} N_{n_{i}}$ $=\infty$ and $N_{n_{i+1}}-N_{n_{i}}>0$. Using (1.2) we get

$$
0<-\delta N_{n_{i}}+P e^{-a N_{n_{i}-k}},
$$

and so

$$
N_{n_{i}}<P / \delta .
$$

Taking the limit of both sides we have $\lim _{i \rightarrow \infty} N_{n_{i}}<\infty$, which contradicts the assumption that $\left\{N_{n}\right\}$ is unbounded. Thus the solution of (1.2) is bounded.

Now we prove the persistence. Assume that

$$
M=\max \left\{N_{-k}, N_{-k+1}, \ldots, N_{0}, N^{*}, P / \delta\right\} .
$$

If $f(x)$ is the function used before, then $f(M)<f\left(N^{*}\right)=0$, and so $(P / \delta) e^{-a M}<M$. Set

$$
F(N)=\frac{P}{\delta} e^{-a N} \quad \text { for } N \in(0, M] .
$$

Clearly $F(N)$ is decreasing on $(0, M]$, and so it has a maximum at $N=0$, and $F(0)=P / \delta \leq M$. Thus $F(N) \leq M$ for all $\mathrm{N} \in(0, M]$. Note that $N_{0} \in(0, M]$ and so

$$
N_{1}=(1-\delta) N_{0}+P e^{-a N_{0}}<N_{0}+\delta\left(\frac{P}{\delta} e^{-a N_{0}}\right)<N_{0}+\delta M<2 M .
$$

It follows by induction that $N_{n}<2 M$ for $n=1,2, \ldots$, which implies $N_{n-k}<2 M$ for $n=k+1, k+2, \ldots$ Finally, note that

$$
N_{n+1}=(1-\delta) N_{n}+P e^{-a N_{n-k}}>P e^{-a N_{n-k}}>P e^{-2 a M} .
$$

Then

$$
P e^{-2 a M}<N_{n}<2 M \quad \text { for } n \geq n_{1}=k+1, k+2, \ldots
$$

Thus every positive solution of (1.2) is bounded and persistent. 
The following theorem provides some sufficient conditions for oscillation of all positive solutions of (1.2) about $N^{*}$.

A solution $\left\{N_{n}\right\}$ of (1.1) is said to oscillate about the equilibrium $N^{*}$ if the differences $N_{n}-N^{*}$ are neither eventually positive nor eventually negative.

Theorem 2.2. Assume that (1.3) holds, and

$$
P a e^{-a N^{*}}>\left(\frac{k}{k+1}\right)^{k+1}(1-\delta)^{k+1} .
$$

Then every solution of (1.2) oscillates about $N^{*}$.

Proof. Assume for contradiction that (1.2) has a solution which does not oscillate about $N^{*}$, and let

$$
N_{n}=N^{*}+\frac{1}{a} z_{n} .
$$

Without loss of generality we assume that $N_{n}>N^{*}$ and this implies that $z_{n}>0$. (The case $N_{n}<N^{*}$ implies that $z_{n}<0$ for which the proof is similar. In fact, we will see below that if $z_{n}$ is a negative solution of (2.5) then $U_{n}=-z_{n}$ is a positive solution of (2.5).) From the transformation (2.4) it is clear that $N_{n}$ oscillates about $N^{*}$ if and only if $z_{n}$ oscillates about zero. The transformation (2.4) turns (1.2) into

$$
z_{n+1}-z_{n}+\delta z_{n}+\operatorname{Pae}^{-a N^{*}}\left(1-e^{-z_{n-k}}\right)=0,
$$

or

$$
z_{n+1}-z_{n}+\delta f_{1}\left(z_{n}\right)+P a e^{-a N^{*}} f_{2}\left(z_{n-k}\right)=0,
$$

where

$$
f_{1}(u)=u, \quad f_{2}(u)=1-e^{-u} .
$$

Note that

$$
u f_{1}(u)>0 \quad \text { for } u \neq 0, \quad \lim _{u \rightarrow 0} \frac{f_{1}(u)}{u}=1,
$$

and

$$
u f_{2}(u)>0 \quad \text { for } u \neq 0, \quad \lim _{u \rightarrow 0} \frac{f_{2}(u)}{u}=1 .
$$

Also we claim that

$$
\begin{array}{ll}
f_{1}(u) \leq u & \text { for } u>0, \\
f_{2}(u) \leq u & \text { for } u>0 .
\end{array}
$$

The proof of (2.8) is simple. The proof of (2.9) follows from the observation that $f_{2}(0)=0$, and that

$$
\frac{d}{d u}\left[f_{2}(u)-u\right]=-\left(1-\frac{1}{e^{u}}\right)<0 \quad \text { for } u>0 .
$$


The linearized equation associated with $(2.5)$ is

$$
y_{n+1}-y_{n}+\delta y_{n}+P a e^{-a N^{*}} y_{n-k}=0,
$$

and every solution of (2.10) oscillates if (2.3) holds (see [9, Lemma 1]). The proof is now an elementary consequence of the linearized oscillation theorem established by Ladas and Qian [10] according to which if (2.6)-(2.9) hold, then every solution of (2.5) oscillates if and only if every solution of (2.10) oscillates. Thus, in conclusion every positive solution of (1.2) oscillates about $N^{*}$.

Now, we study the global asymptotic stability of $N^{*}$ for $(1.2)$ when $k=1$.

The equilibrium $N^{*}$ of (1.2) is called stable if for every $\varepsilon>0$, there exists $\delta>0$ such that if $N_{-1}, N_{0} \in I$ and $\left|N_{0}-N^{*}\right|+\left|N_{-1}-N^{*}\right|<\delta$, then $\left|N_{n}-N^{*}\right|<\varepsilon$ for all $n \geq-1$.

The equilibrium $N^{*}$ of (1.2) is called locally asymptotically stable if it is stable and there exists $\gamma>0$ such that if $N_{-1}, N_{0} \in I$ and $\left|N_{0}-N^{*}\right|+$ $\left|N_{-1}-N^{*}\right|<\gamma$, then

$$
\lim _{n \rightarrow \infty} N_{n}=N^{*}
$$

We say that $N^{*}$ is a global attractor if for every $N_{-1}, N_{0} \in I$ we have $\lim _{n \rightarrow \infty} N_{n}=N^{*}$. Further, $N^{*}$ is called globally asymptotically stable if $N^{*}$ is stable and also a global attractor.

A solution $\left\{N_{n}\right\}$ of (1.2) is said to be periodic of prime period $a$, or of minimal period $a$, if it is periodic of period $a$, where $a$ is the least positive integer for which $N_{n+a}=N_{n}$ for $n=0,1, \ldots$

Let $f$ be a $C^{3}$ function in some interval $I$. The Schwarzian derivative $S f(x)$ of $f$ at a point $x \in I$, where $f^{\prime}(x) \neq 0$, is given by

$$
S f(x)=\frac{f^{\prime \prime \prime}(x)}{f^{\prime}(x)}-\frac{3}{2}\left(\frac{f^{\prime \prime}(x)}{f^{\prime}(x)}\right)^{2} .
$$

Theorem 2.3. Assume that (1.3) holds, $k=1$ and

$$
P a e^{-a N^{*}}<\delta \text {. }
$$

Then the unique steady state $N^{*}$ of (1.2) is locally asymptotically stable.

Proof. It suffices to prove that the zero solution of the linearized equation of (1.2) is locally asymptotically stable. The linearized equation associated with (1.2) when $k=1$ is

$$
y_{n+1}-(1-\delta) y_{n}+P a e^{-a N^{*}} y_{n-1}=0,
$$

and the difference equation (2.12) is asymptotically stable if (2.11) holds (see $[3$, Theorem A]).

Now, we will give sufficient conditions for $N^{*}$ to be globally asymptotically stable. 
Theorem 2.4. Assume that (1.3) and (2.11) hold, with $N_{-1}, N_{0}>0$. Then $N^{*}$ is globally asymptotically stable.

Proof. When $k=1,(1.2)$ can be rewritten in the form

$$
N_{n+1}=f\left(N_{n}, N_{n-1}\right)=\alpha N_{n}+P e^{-a N_{n-1}},
$$

with $N_{-1}, N_{0}>0$. In Theorem 2.3 we saw that $N^{*}$ is locally asymptotically stable. To prove that $N^{*}$ is globally asymptotically stable we will prove that $N^{*}$ is a global attractor of all positive solutions of (2.13). To prove this we will apply Theorem 2.3 of [6]. In our case

$$
\begin{aligned}
f(u, v) & =\alpha u+P e^{-a v}, \\
F(v) & =f\left(f\left(N^{*}, v\right), v\right)=\alpha^{2} N^{*}+(\alpha P+P) e^{-a v},
\end{aligned}
$$

and $\alpha=1-\delta>0$. Note that

$\left(\mathrm{h}_{1}\right) f \in C[(0, \infty) \times(0, \infty),(0, \infty)]$,

$\left(\mathrm{h}_{2}\right) f(u, v)$ is nondecreasing in $u$ and decreasing in $v$,

$\left(\mathrm{h}_{3}\right) f(u, v) / u$ is nonincreasing in $u$,

$\left(\mathrm{h}_{4}\right)(2.13)$ has a unique steady state $N^{*}$.

It remains to prove that $F$ has no periodic points of prime period 2 . Since $F\left(N^{*}\right)=N^{*}$, in view of Lemma A of [6] it is sufficient to show that all solutions of the difference equation

$$
y_{n+1}=F\left(y_{n}\right), \quad n=0,1, \ldots,
$$

converge to $N^{*}$. This will be accomplished by showing that the Schwarzian derivative of $F$ is negative together with the observation that because of (2.11) the equilibrium $N^{*}$ of (2.14) is locally asymptotically stable. To this end observe that

$$
S F(v)=-\frac{1}{2} a^{2}<0 .
$$

The proof is now an elementary consequence of [6, Theorem 2.3] according to which if $\left(\mathrm{h}_{1}\right)-\left(\mathrm{h}_{4}\right)$ hold, and the function $F(v)=f\left(f\left(N^{*}, v\right), v\right)$ has no periodic points of prime period 2 , then $N^{*}$ is globally asymptotically stable.

3. Nonautonomous case. In this section we study the oscillation behavior of all positive solutions of (1.5). A solution $\left\{N_{n}\right\}$ of (1.5) is said to oscillate about the sequence $\left\{N_{n}^{*}\right\}$ if the differences $N_{n}-N_{n}^{*}$ are neither eventually positive nor eventually negative.

THEOREM 3.1. Let $\left\{N_{n}^{*}\right\}$ be a positive solution of (1.5), and assume that (1.6) holds. Then every positive solution of (1.5) oscillates about $\left\{N_{n}^{*}\right\}$ 
if and only if every solution of the linear equation

$$
y_{n+1}-y_{n}+\delta_{n} y_{n}+P_{n} a e^{-a N^{*}} y_{n-k}=0
$$

oscillates.

Proof. Let $\left\{N_{n}\right\}$ be a positive solution of (1.5). Set

$$
N_{n}=N_{n}^{*}+\frac{1}{a} z_{n}
$$

From the transformation (3.2) it is clear that $N_{n}$ oscillates about $N^{*}$ if and only if $z_{n}$ oscillates about zero. The transformation (3.2) turns (1.5) into

$$
z_{n+1}-z_{n}+\delta_{n} z_{n}+P_{n} a e^{-a N^{*}}\left(1-e^{-z_{n-k}}\right)=0,
$$

or

$$
z_{n+1}-z_{n}+\delta_{n} f_{1}\left(z_{n}\right)+P_{n} a e^{-a N^{*}} f_{2}\left(z_{n-k}\right)=0,
$$

where

$$
f_{1}(u)=u, \quad f_{2}(u)=1-e^{-u} .
$$

Note that

$$
u f_{1}(u)>0 \quad \text { for } u \neq 0, \quad \lim _{u \rightarrow 0} \frac{f_{1}(u)}{u}=1,
$$

and

$$
u f_{2}(u)>0 \quad \text { for } u \neq 0, \quad \lim _{u \rightarrow 0} \frac{f_{2}(u)}{u}=1 .
$$

Also we claim that

$$
\begin{array}{ll}
f_{1}(u) \leq u & \text { for } u>0 \\
f_{2}(u) \leq u & \text { for } u>0 .
\end{array}
$$

The proof of (3.6) is simple. The proof of (3.7) follows from the observation that $f_{2}(0)=0$ and that

$$
\frac{d}{d u}\left[f_{2}(u)-u\right]=-\left(1-\frac{1}{e^{u}}\right)<0 \quad \text { for } u>0 .
$$

The proof is now an elementary consequence of [10, Theorem 3] according to which if (3.4)-(3.7) hold then every solution of (1.5) oscillates if and only if every solution of (3.1) oscillates.

REMARK 3.1. Theorem 3.1 shows that the oscillation of (3.1) implies the oscillation of (1.5). Therefore we can use the results of [13] to obtain oscillation criteria for (1.5). The details are left to the reader.

Acknowledgements. The authors would like to thank the referee for his (her) helpful suggestions that improved the presentation of the results of this manuscript. 


\section{References}

[1] S. Elaydi, Global stability of nonlinear difference equations, J. Differ. Equations Appl. 2 (1996), 87-96.

[2] R. DeVault, E. A. Grove, G. Ladas, R. Levins and C. Puccia, Oscillation and stability in a delay model of a perennial grass, in: Proc. Dynamic Systems and Applications (Atlanta, 1993), Dynamic Publ., 1994, 87-94.

[3] R. DeVault, W. Kosmala, G. Ladas and S. W. Schultz, Global behavior of $y_{n+1}=$ $\frac{p+y_{n-k}}{q y_{n}+y_{n-k}}$, Nonlinear Anal. 47 (2001), 4743-4751.

[4] I. Györi and G. Ladas, Oscillation Theory of Delay Differential Equations with Applications, Clarendon Press, Oxford, 1991.

[5] V. Hutson and K. Schmitt, Persistence and the dynamics of biological systems, Math. Biosci. 111 (1992), 1-71.

[6] V. L. Kocic and G. Ladas, Global attractivity in a nonlinear second-order difference equation, Comm. Pure Appl. Math. 48 (1995), 1115-1122.

[7] -, -, Global attractivity in nonlinear delay difference equations, Proc. Amer. Math. Soc. 115 (1992), 1083-1088.

[8] —, - Global Behavior of Nonlinear Difference Equations of Higher Order, Kluwer, Dordrecht, 1993.

[9] -, - Oscillation and global attractivity in a discrete model of Nicholson's blowflies, Appl. Anal. 38 (1990), 21-31.

[10] G. Ladas and C. Qian, Linearized oscillations for nonautonomous delay difference equations, in: Contemp. Math. 129, Amer. Math. Soc., 1992, 115-125.

[11] P. Liu and X. Cui, A discrete model of competition, Math. Comput. Simulation 49 (1999), 1-12.

[12] S. H. Saker and S. Agarwal, Oscillation and global attractivity of a periodic survival red blood cells model, J. Dynam. Cont. Discrete and Impulsive Systems B Appl. Algorithms, to appear.

[13] X. H. Tang and J. S. Yu, Oscillation of delay difference equation, Comput. Math. Appl. 37 (1999), 11-20.

[14] M. Ważewska-Czyżewska and A. Lasota, Mathematical problems of the dynamics of the red blood cells system, Mat. Stos. 6 (1976), 23-40.

[15] R. Y. Zhang and Z. C. Wang, Periodic solutions of a single species discrete population model with periodic harvest/stock, Comput. Math. Appl. 39 (2000), 77-90.

[16] Z. Zhou and Q. Zhang, Global attractivity of a nonautonomous logistic difference equation with delay, ibid. 38 (1999), 57-64.

Faculty of Mathematics and Computer Science

Adam Mickiewicz University

Umultowska 87

61-614 Poznań, Poland

E-mail: kuba@amu.edu.pl
Department of Mathematics Faculty of Science Mansoura University Mansoura, 35516, Egypt

E-mail: shsaker@mum.mans.eun.eg

Received on 5.9.2002;

revised version on 31.3 .2003 